\title{
Research on Error Ratio Calculator for the Electric Energy Meter Error Calibrator Device
}

\author{
Jun Chen ${ }^{1, a}$, Yu Guo ${ }^{1, b}$ and Yinglong Chen ${ }^{2, c}$ \\ ${ }^{1}$ State Grid HuBei Electric Power Research Institute, HuBei Wuhan 430077, China \\ ${ }^{2}$ Materials Branch of State Grid East Inner Mongolia Electric Power Company Limited, Inner \\ Mongolia Autonomous Region Hohhot, 010000, China \\ achenjun1964@sohu.com, bgy051901010206@163.com
}

Keywords: error calibration, pulse number, error ratio calculator.

Abstract. With the rapid development of national economy, electric energy which is considered to be an important energy source has obtained wide application in all sectors of society. Electric energy meter is regarded as the main instrument in current measurement and economic settlement. Its accuracy has a direct relationship with the behalf between country and meter user. Therefore, it is important to calibrate the error of electric energy meter. At present, the error calibration method remain lag behind relatively, and the level of automation is low. This paper proposed an error ratio calculator which avoids the manual calculation of the traditional calibration pattern and improves the automatic and intelligent level of electric energy meter calibration.

\section{Introduction}

With the extensive application and batch installation of intelligent watt-hour meter, a large number of pipeline watt-hour meter verification systems are emerged, which can detect watt-hour meter massive in a wide range, but the scale testing is not suitable for detecting single watt-hour meter ${ }^{[1]}$. If the pipeline device test a single watt-hour meter randomly, it would waste resources, so a kind of "self-help" type of watt-hour meter error calibration device is required to test error anytime and anywhere. Among them, the error calculator is a vital part of self-service error calibration device ${ }^{[2]}$.

The watt-hour meter calibration is defined as the watt-hour meter is applied with a certain voltage and current, then comparing the measured energy value with the actual consumption of energy value to calculate the error of the watt-hour meter.The means of watt-hour meter calibration is divided into the watt-second method and the standard meter method, owing to the standard meter method realize a direct comparison of the detected energy and the standard energy, the impact on energy error of other influence-quantities are greatly reduced, so it has been widely used.

The majority of traditional watt-hour meter error calibration belong to error comparison of electronic watt-hour meter which is commonly used in high-frequency pulses preset method ${ }^{[3,4]}$. In other words, in the case of the standard meter and the detected meter are continuous operation ${ }^{[5]}$, recording high-frequency pulse number $\mathrm{m}$ of standard output when the detected meter output $\mathrm{N}$ low-frequency pulse, using the $\mathrm{m}$ as a real measuring number of pulses ${ }^{[6]}$, then comparing with high-frequency pulses preset number $\mathrm{m}_{0}$ to calculate the relative error. $\mathrm{m}_{0}$ must be preset in a standard meter or error calculator through the manual calculation on the basis of the various detected meter, then verify the meter, which is prone to miscalculation ${ }^{[7]}$. The calculator described in this article is controlled by software, directly input the various parameters related to error, which can check the error of watt-hour meter.

\section{Function and Main Technical Indicators}

According to the relevant parameter settings, the calculator in this article calculate the error of watt-hour meter by software, its main functions and technical indicators are as follows:

(1) Apply to error comparison of all electronic watt-hour meter;

(2) The number of pulses can be illustrated by the detected meter and the standard meter sent separately; 
(3) The input pulse frequency and voltage range of rectangular wave are allowed within $300 \mathrm{kHz}$ and 3.5 24V respectively;

(4) With digital keys 0-9 for entering a number;

(5) The number(N) of the detected meter's low-frequency pulse within the range of 0 to 9999;

(6) The transformation ratio's $\left(\mathrm{K}_{\mathrm{U}}, \mathrm{K}_{\mathrm{I}}\right)$ setting by the PT and CT of standard meter;

(7) The frequency pulse constant's(CHO) setting of standard meter ;

(8) The low frequency pulse constant's(CL) setting of detected meter;

(9) The indication range of error is $\pm 99.999 \%$.

\section{Hardware Structure}

The program uses the $8031 \mathrm{MCU}$ of MCS-51 series, which is an early Intel's mature product, with high integration, strong processing capabilities, high reliability, simple structure, low price and rich shared resources and a series of advantages.

Using $8031 \mathrm{MCU}$ as a central processing unit, the pulse signal is sent to the error calculator, at the same time, the error calculator receives the standard pulse signal from the standard meter, processing the calculation error of the detected meter and the standard meter time-sharing, and displays the error value of the detected meter.

Hardware schematic block diagram shown in figure $1, \mathrm{~T}_{0}$ and $\mathrm{T}_{1}$ are as counter mode to add up the pulse sent by the detected meter and the standard meter respectively. P0 port is used for transmitting data, 2764 is work program area, 6264 is external data memory unit, 373 is lower address latch.

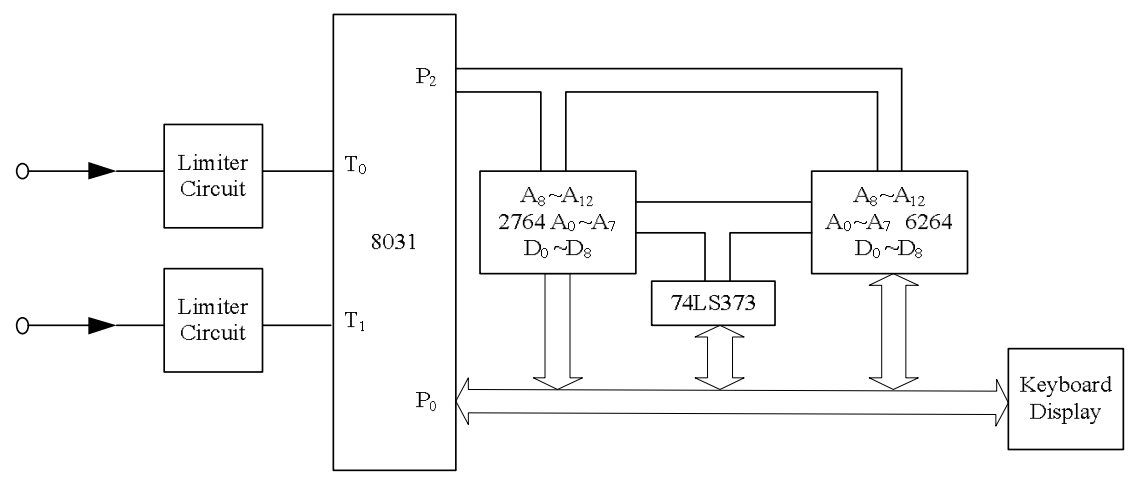

Fig.1 The hardware principle diagram

\section{Working Principle}

In the case of the standard meter and the detected meter are continuous work, using the the number of pulses output by the detected meter to control the count of the standard watt-hour meter so that it can determine the relative error of the detected meter. The counted number of pulses must be preset to the standard tables or the error calculator by the manual calculation on the basis of the various detected meter, this process is prone to error. The error calculator is used for inputting the pulse value under various loads of the standard single-phase watt-hour meter and the detected single-phase watt-hour meter, and then calculate the error of the detected single-phase watt-hour meter by comparing.

After a limiter circuit, the pulse of the standard meter and the detected meter are connected with the $\mathrm{T}_{1}$ and $\mathrm{T}_{0}$ counter of $8031 \mathrm{MCU}$, respectively. Using the number keys and function keys input the frequency pulse constant $\mathrm{C}_{\mathrm{HO}}$, the low frequency pulse constant $\mathrm{C}_{\mathrm{L}}$ of the detected meter, the external current and voltage transformer's transformation ratio $\mathrm{K}_{\mathrm{I}}$ and $\mathrm{K}_{\mathrm{U}}$ of the standard meter. When there are no external current and voltage transformers, $\mathrm{K}_{\mathrm{I}}$ and $\mathrm{K}_{\mathrm{U}}$ 's value are set to 1. Finally, inputting the low frequency pulse number $\mathrm{N}$ of the detected meter(select the value of $\mathrm{N}$ appropriately so that the pulse number of the standard meter accumulated by the calculator is not less than the minimum cumulative number of all levels watt-hour meter's requirement), then compare the error. Stopping counting when the counter T0 count to $\mathrm{N}$, the actual cumulative number of the standard meter counted by counter $\mathrm{T} 1$ is set to $\mathrm{m}$. 
Then the results are as follows:

The electric energy value of the detected meter is:

$$
W=\frac{N}{C_{L}}(k \mathrm{~W} \bullet \mathrm{h})
$$

The electric energy value of the standard meter is:

$$
W_{0}=\frac{m}{C_{\mathrm{HO}}} k_{\mathrm{I}} \bullet k_{\mathrm{U}}(k \mathrm{~W} \bullet \mathrm{h})
$$

The relative error of the detected meter is:

$$
\gamma=\frac{W-W_{0}}{W_{0}} \times 100 \%
$$

Substituting Equation (1), (2) into (3) to obtain the relative error of the detected meter:

$$
\gamma=\frac{\frac{N}{C_{\mathrm{L}}}-\frac{m}{C_{\mathrm{HO}}} k_{\mathrm{I}} k_{\mathrm{U}}}{\frac{m}{C_{\mathrm{HO}}} k_{\mathrm{I}} k_{\mathrm{U}}}=\frac{C_{\mathrm{HO}} N-C_{\mathrm{L}} m k_{\mathrm{I}} k_{\mathrm{U}}}{C_{\mathrm{L}} m k_{\mathrm{I}} k_{\mathrm{U}}} \times 100 \%
$$

\section{Design of Software}

The control of error calculator is implemented by the upper computer software, the software needs to be able to set the host computer to communicate with the error calculator to achieve the control purpose. The main task of the software to communicate with the error calculator is the relevant parameters for counting are sent to the error calculator by host computer, so as to the calculator can count the watt-hour meter error in accordance with the prescribed formulas, the error calculator also requires to send the error value to the host computer, so the host computer can be kept in the database for future review and analysis.

The work procedure mainly complete the following work:

(1)According to the requirement of counting, designing the mode of inputting data in accordance with the function and numeric keys;

(2) Controlling the pulses cumulative number of the detected meter and the standard meter;

(3) Data processing, that is data calculation and error illustration on the basis of equation (4).

The main program flow chart shown in figure 2. Starting work program after the system switch on. First, carrying out system initialization, setting the work mode and interrupt priority of $\mathrm{T}_{0}$ and $\mathrm{T}_{1}$. Then wait a moment and input relative parameters $\mathrm{K}_{U}, \mathrm{~K}_{\mathrm{I}}, \mathrm{C}_{\mathrm{L}}, \mathrm{C}_{\mathrm{HO}}$ and $\mathrm{N}$ by the keyboard. Finally, starting the timing counter $T_{0}$ and $T_{1}$ to count, $T_{0}$ and $T_{1}$ 's interruption are allowed. During $\mathrm{T}_{0}$ 's interruption, tuning out the subroutine that demonstrate the pulses cumulative number of the standard meter or the detected meter. While $\mathrm{T}_{0}$ port's counting value reaches $\mathrm{N}$, stopping $\mathrm{T}_{0}$ and $\mathrm{T}_{1}$ from counting, Substituting $T_{1}$ port's accumulated value $m$ to equation (4), then show the relative error of detected meter.

\section{Conclusion}

The error calculator designed in this paper avoids the manual calculation process of traditional calibration work, Making error checking work more simple, easy, and easy to grasp, the exploitation and application of the calculator will improve watt-hour meter error calibration's work efficiency and automation level meter.

The calculator is simple structure, easy to manufacture, low cost, stable and reliable, will use in self-service watt-hour meter error correction device, so that self-service watt-hour meter error 
correction device can conduct watt-hour meter's fully automatic error calibration, greatly reduce the device's production costs, and increase the accuracy of error calibration. The calculator solves the inconvenient technical problems of individual single-phase watt-hour meter's error testing caused by pipeline, and also settle the residential users to participate in the supervised matter of intelligent watt-hour meter smart meter's measurement accuracy, making energy calculating more fairly.

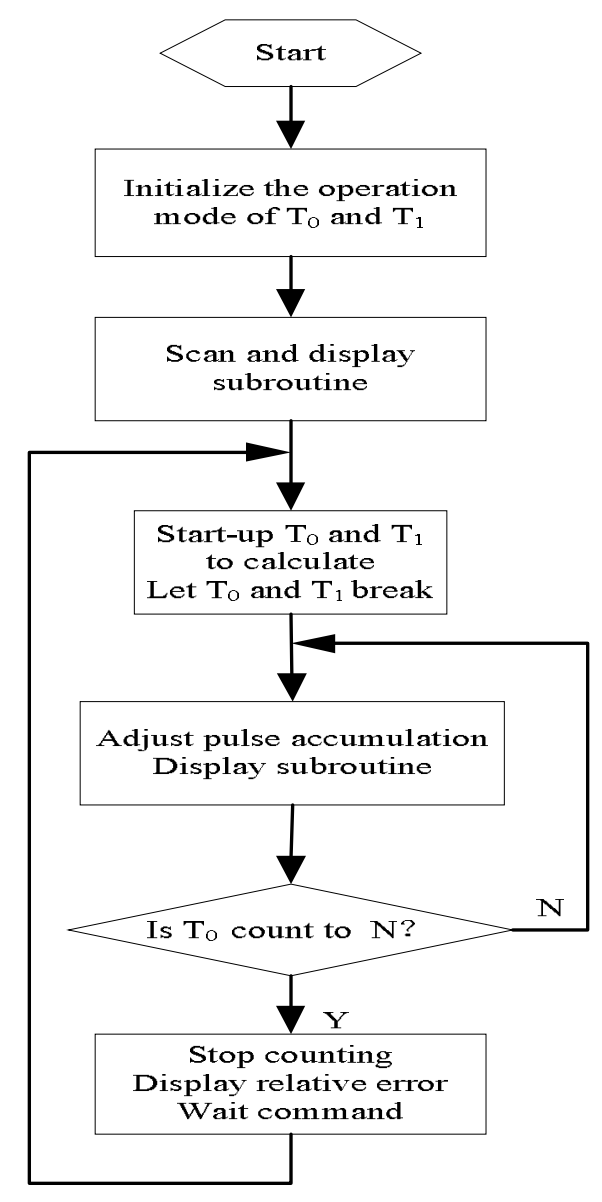

Fig.2 The flow chart of the main program

\section{References}

[1] Jun Chen, Yinglong Chen, Yu Guo, Sike Wang. Electrical Measurement \& Instrumentation, 108-111( 2014), In Chinese.

[2] Mei Zhang, Jianrong Cao, Chengxu Duan. Electric Power Automation Equipment, 59-61(2004), In Chinese.

[3] He Wen, Liangzhang Zhou, Zhao-sheng Teng, Siyu Guo, Xiaoqing Li. JOURNAL OF EEE, 83-85(2013), In Chinese.

[4] Qingsong Zhao. Harbin University of Science and Technology, (2009), In Chinese.

[5] Hui Yuan, Dongmei Yao, Chaojun Zhang. CHINA MEASUREMENT \& TEST,20-22(2012), In Chinese.

[6] Huayun Yang, AI Bing, Bo Jiang, Xianshun Chen, Yongbo Yang. Electrical Measurement \& Instrumentation, 53-57(2013), In Chinese.

[7] Weiming Sun ,Guoying Lin. Electrical Measurement \& Instrumentation, 13-15(2010), In Chinese. 